\title{
Bending of Thin Elastic Plates Containing Two Circular Holes
}

\author{
Kuang-Chong Wu, Tsungyen Tsai \\ Institute of Applied Mechanics, National Taiwan University \\ Taipei, Taiwan \\ wukciam@ntu.edu.tw
}

\section{Extended Abstract}

The problem of a thin elastic plate containing holes subjected to bending or twisting is important in engineering applications because of the stress concentrations at the edge of the holes. Goodier [1] investigated the influence of circular and elliptic holes on the transverse flexure of isotropic elastic plates in various loading cases. Lekhnitskii [2] developed a complex variable method for anisotropic plates and provided explicit solutions for a circular hole in an orthotropic plate. Based on Lekhnitskii's method, Hsieh and Hwu [3] developed a Stroh-like formalism and derived analytical solutions for certain anisotropic plates. Exact solution for general anisotropic materials under arbitrary uniform loading conditions was derived by $\mathrm{Wu}$ and Hsiao [4]. Explicit expressions for the deflection and moments on the edge of an elliptic hole in an orthotropic plate subjected to bending or twisting moments were obtained. There are very few studies on interaction of multiple holes in infinite anisotropic plates under bending. In this work a new boundary integral equation [5] is used to analyze an infinite anisotropic plate containing two circular holes to investigate the interactions of the holes.

Specifically the moments at the edge of the holes were calculated for two circular holes with identical radius $a$ and center-to-center distance $d$ subjected to a remote uniform moment. The thickness was assumed as $3 \mathrm{~mm}$. T300 Carbon/5208Epoxy with $E_{1}=181 \mathrm{GPa} 、 E_{2}=10.3 \mathrm{GPa} 、 G_{12}=7.17 \mathrm{GPa} 、 v_{12}=0.28$ was considered such that $D_{11}=409 、 D_{12}=23.3 、 D_{22}=6.52 、 D_{66}=16.1\left(\mathrm{GPa} \cdot \mathrm{mm}^{3}\right)$; An isotropic material with $E=300 \mathrm{GPa}, v=0.3$ was also considered. It is found that the interactions of the holes are insignificant except in the regions between the holes when the holes are close.

\section{References}

[1] J. N. Goodier, "The influence of circular and elliptical holes on the transverse flexure of elastic plates," Phil. Mag., vol. 22, no. 69-80, 1936.

[2] S. G. Lekhnitskii. Anisotropic plates. New York: Gordon and Breach, 1968.

[3] M.-C. Hsieh, and C. Hwu, "Anisotropic elastic plates with holes/cracks/inclusions subjected to out-of-plane bending moments," Int. J. Solids Struct., vol. 39, no. 19, pp. 4905-4925, 2002.

[4] K.-C. Wu and P.-S. Hsiao, „An exact solution for an anisotropic plate with an elliptic hole under arbitrary remote uniform moments," Composites Part B, vol. 75, pp. 281-287, 2015.

[5] K.-C. Wu and P.-S. Hsiao, „A new boundary integral formulation for bending of anisotropic plates,” Acta Mech., vol. 228, pp. 2741-2750, 2017. 